\title{
Elevated p-CREB-2 (ser 245) expression is potentially associated with carcinogenesis and development of breast carcinoma
}

\author{
CHUI-FENG FAN ${ }^{1,2}$, XIAO-YUN MAO ${ }^{3}$ and EN-HUA WANG ${ }^{1,2}$ \\ ${ }^{1}$ Department of Pathology; ${ }^{2}$ Institute of Pathology and Pathophysiology; ${ }^{3}$ Department of Breast Surgery, \\ and Department of Surgical Oncology, Research Unit of General Surgery, The First Affiliated Hospital and \\ College of Basic Medical Sciences of China Medical University, Shenyang 110001, P.R. China
}

Received August 3, 2011; Accepted October 19, 2011

DOI: $10.3892 / \mathrm{mmr} .2011 .657$

\begin{abstract}
CREB-2, also known as ATF-4, belongs to the CREB proteins, a family of transcription factors phosphorylated at serine residues by protein kinase A (PKA). This family is known to stimulate the transcription of genes containing CRE elements. Recently, some studies have demonstrated elevated CREB-2 expression in certain tumor types, including breast carcinoma, compared to their corresponding non-tumor tissues. However, the expression and clinical significance in malignant tumors, including breast carcinoma, of p-CREB-2 (ser 245), a phosphorylated form of the CREB-2 protein at serine 245 site, which is believed to be an active type of this protein, have not been clearly documented. In the present study, we investigated the expression of p-CREB-2 (ser 245) in a group of tumor and non-tumor breast tissues, including normal breast epithelia, hyperplasia, dysplasia, carcinoma in situ and infiltrating carcinoma of the breast using tissue microarray and immunohistochemistry (IHC). p-CREB-2 (ser 245) immunostaining was detected in the nucleus and cytoplasm of these tissues. Compared to normal breast epithelia and breast hyperplasia (total positive rate $13.3 \%$ ), there was increased expression of p-CREB-2 (ser 245) in dysplasia, carcinoma in situ (total positive rate 35.7\%) and infiltrating carcinoma of the breast (total positive rate $60.0 \%)(\mathrm{p}<0.05)$. The highest expression of p-CREB-2 (ser 245) was found in infiltrating breast carcinoma (total positive rate 60\%) compared to normal breast epithelia and all types of non-infiltrating lesions (total positive rate $27.6 \%)(\mathrm{p}<0.05)$. In addition, increased expression of p-CREB-2 (ser 245) was found to be associated with lymph node metastasis in infiltrating breast carcinoma $(p<0.05)$. Immunofluorescent staining confirmed stronger staining of p-CREB-2 (ser 245) in breast cancer MCF 7 and MDA-MB231 cells compared with normal breast epithelial MCF 10A
\end{abstract}

Correspondence to: Dr En-Hua Wang, Department of Pathology, First Affiliated Hospital and College of Basic Medical Sciences of China Medical University, Shenyang 110001, P.R. China

E-mail:wangenh@hotmail.com

Key words: breast carcinoma, CREB-2, dysplasia, hyperplasia, immunohistochemistry, tissue microarray cells. Western blotting revealed elevated expression levels of p-CREB-2 (ser 245) in 17 cases of breast carcinoma compared with corresponding normal breast tissues $(p<0.05)$. These results indicate that elevated expression of p-CREB-2 (ser 245) may potentially contribute to carcinogenesis and cancer development of breast carcinoma.

\section{Introduction}

Breast cancer contributes to a large proportion of the total cancer incidence among females worldwide. Moreover, the incidence and mortality of breast cancer have also climbed sharply in China during the last two decades. An understanding of the precise molecular mechanisms of breast carcinogenesis is essential for the discovery of promising prognostic markers, predictors of response to therapy and novel targeted agents.

The CREB family was originally characterized as a group of factors that are responsive to increased intracellular levels of cyclic AMP, although their functions in cellular signaling have not been consistent among all members of this family (1). Recently, CREB-2, a member of this family, was found to be upregulated in certain types of malignant tumors, including breast carcinoma, particularly near necrotic regions compared with their non-tumor counterparts (2). There is increasing evidence that upregulation of CREB-2 may be involved and play critical roles in the unfolded protein response (UPR), which is activated and confers a survival advantage for tumor cells under hypoxia (3-5). Phosphorylation of CREB-2 at serine 245 has been linked to its activation, rendering a critical role for CREB-2 in skeletal formation (6). Hogan et al reported that phosphorylation of CREB-2 may lead to a significant increase in CRE-reporter activation (7). Although studies present evidence of the upregulation of CREB-2 in some malignant tumors, including breast carcinoma, the expression of this activated form, namely p-CREB-2 (ser 245), and its clinical significance in breast carcinoma is not yet clear. In the present study, we investigated p-CREB-2 (ser 245) expression in a group of tumor and non-tumor breast tissues including normal breast epithelia, hyperplasia, dysplasia, carcinoma in situ and infiltrating carcinoma of the breast to elucidate its possible impact on carcinogenesis of breast cancer and its potential clinical significance in this type of malignant tumor. 


\section{Materials and methods}

Tissue samples. Expression levels of p-CREB-2 (ser 245) were investigated using immunohistochemical analysis of commercially available tissue microarrays (TMAs) from Chaoying Biotechnology Co., Ltd (Shanxi, China), which contained a total of 101 tissue samples of tumor or non-tumor breast tissues from 49 patients. Fresh tumor specimens from 17 patients with breast carcinoma were obtained following surgical resection at the First Affiliated Hospital of China Medical University. Tumor and the paired non-tumor portion of the same case were quickly frozen in liquid nitrogen and maintained at $-70^{\circ} \mathrm{C}$. The histological types were confirmed by at least two pathologists following the Criteria for the Classification of Breast Cancer by the World Health Organization (8). The tumors were classified according to the tumor-node-metastasis (TNM) stage revised by the National Comprehensive Cancer Network (NCCN) in 2010 (9). The histological types included normal breast epithelia, hyperplasia, dysplasia, carcinoma in situ and infiltrating breast carcinoma, which are outlined in Table I. None of the patients had received chemotherapy or radiotherapy prior to tumor excision. Patient ages at the time of surgery ranged from 15 to 69 years, with an average age of 43.7. The utilization of the tumor material for research purposes was approved by regional ethics committees in Xi'an and Shenyang, China. Informed consent was obtained from all enrolled patients prior to surgery.

Immunohistochemical staining. Immunohistochemistry (IHC) assay for p-CREB-2 (ser 245) expression was performed on TMA sections using the peroxidase-labeled streptavidin-biotin method. Immunohistochemical staining was performed using UltraSensitive $^{\mathrm{TM}}$ S-P kits (Maixin Biotechnology, FuZhou, Fujian, China) according to the manufacturer's instructions. TMA sections were deparaffinized and hydrated in sequential treatments of xylene, ethanol and water. Citrate buffer (0.01 M citric acid, $\mathrm{pH}$ 6.0) was used to retrieve antigens in a heated pressure cooker. Endogenous peroxidase activity and non-specific binding were blocked with $3 \% \mathrm{H}_{2} \mathrm{O}_{2}$ and nonimmune sera, respectively. Sections were then incubated with primary rabbit anti-human p-CREB-2 (ser 245) antibody (Santa Cruz, USA; dilution: 1:50) overnight at $4^{\circ} \mathrm{C}$. The following day biotinylated secondary antibody and streptavidin-horseradish peroxidase (HRP) (Maixin Biotechnology) were added. The peroxidase reaction was developed with 3,3'-diaminobenzidine tetrahydrochloride (Maixin Biotechnology). Counterstaining was performed lightly with hematoxylin and the sections were dehydrated in alcohol prior to mounting. For the negative control, phosphate-buffered saline (PBS) was used in place of the primary antibodies.

Immunohistochemical evaluation. The expression pattern of p-CREB-2 (ser 245) was evaluated by at least two investigators independently from each other, who had no knowledge of the patients' clinical status. Cases with discrepancies were jointly re-evaluated by the investigators and a consensus was obtained. IHC scoring was based on two parameters: the proportion of immunopositive cells and their intensity of immunoreactivity. The proportion of immunopositive cells was categorized as follows: $0,<10 \% ; 1, \geq 10$ to $<25 \% ; 2, \geq 25$ to
$<50 \% ; 3, \geq 50$ to $<75 \%$; and $4, \geq 75 \%$. The staining intensity was categorized by relative intensity as follows: 0 , no positivity; 1 , weak; 2 , moderate; and 3 , strong. A final immunoreactivity score of each sample was obtained by multiplying the two individual scores. To obtain statistical results, a final score less than 2 was considered to be negative, while scores of 2 or more were considered as positive..

Cell culture. Human breast epithelial cell line MCF 10A, and breast carcinoma cell lines MCF 7 and MDA-MB-231, were cultured in Dulbecco's modified Eagle's medium (DMEM) and L15 tissue culture medium, containing 10\% fetal calf serum (Invitrogen, Carlsbad, CA, USA), $100 \mathrm{IU} / \mathrm{ml}$ penicillin (Sigma, St. Louis, MO, USA) and $100 \mu \mathrm{g} / \mathrm{ml}$ streptomycin (Sigma) at $37^{\circ} \mathrm{C}$ in a humidified atmosphere $\left(5 \% \mathrm{CO}_{2}, 95 \%\right.$ air $)$.

Immunofluorescent staining. Cells were fixed with $4 \%$ paraformaldehyde, followed by blocking with $1 \%$ bovine serum albumin (BSA). They were then incubated with p-CREB-2 (ser 245) polyclonal antibody (dilution 1:50) overnight at $4^{\circ} \mathrm{C}$. The primary antibody was followed by incubation with a secondary antibody conjugated to fluorescein isothicyanate (FITC)-labelled IgG. The nuclei were counterstained with 4',6-diamidino-2-phenylindole (DAPI).

Western blotting. Breast carcinoma and normal breast tissues were lysed in 10 volumes (w/v) of lysis buffer. Following centrifugation, the supernatant was collected and quantified. The same amount of total protein was separated by $10 \%$ sodium dodecyl sulfate polyacrylamide gel electrophoresis (SDS-PAGE) and then transferred to a polyvinylidene fluoride (PVDF) membrane. The membrane was then incubated overnight at $4^{\circ} \mathrm{C}$ with antibodies against p-CREB-2 (ser 245) (sc-1188, 1:500; Santa Cruz) and $\beta$-actin (sc-130301, 1:200; Santa Cruz). Following incubation with the secondary antibody labeled with HRP at room temperature for $2 \mathrm{~h}$, protein bands were visualized using enhanced chemiluminescence (ECL) and detected using the BioImaging System.

Statistical analysis. All statistical analyses were performed using SPSS 13.0 for Windows (SPSS Inc., Chicago, IL, USA). The Pearson's Chi-square test was used to analyze the relationship between p-CREB-2 (ser 245) expression and clinicopathological factors. The McNemar's test was used to compare the p-CREB-2 (ser 245) expression in tumor and several types of non-tumor breast tissues. The t-test was used to analyze the results of Western blotting. $\mathrm{P}<0.05$ was considered to be statistically significant.

\section{Results}

Expression of p-CREB-2 (ser 245) in tumor and non-tumor breast tissues. Immunostaining of p-CREB-2 (ser 245) in tumor and non-tumor breast tissues is shown in Fig. 1. p-CREB-2 (ser 245) immunostaining was detected in the nuclei and cytoplasm. The positive rates of p-CREB-2 (ser 245) expression in these tissues are outlined in Table I. The positive rates of p-CREB-2 (ser 245) expression in normal breast epithelia and hyperplasia were $14.29 \%(1 / 7)$ and $12.50 \%$ (1/8), respectively, which showed no significant 
Table I. Positive rate of p-CREB-2 (ser 245) expression in tumor and non-tumor breast tissues.

\begin{tabular}{|c|c|c|c|c|c|c|c|c|c|}
\hline & \multicolumn{8}{|c|}{ Histological type } & \multirow[b]{2}{*}{ Total } \\
\hline & $\begin{array}{c}\text { Normal } \\
\text { epithelia }\end{array}$ & Hyperplasia & $\begin{array}{c}\text { Mild } \\
\text { dysplasia }\end{array}$ & $\begin{array}{l}\text { Moderate } \\
\text { dysplasia }\end{array}$ & $\begin{array}{c}\text { Severe } \\
\text { dysplasia }\end{array}$ & $\begin{array}{l}\text { Carcinoma } \\
\text { in situ }\end{array}$ & $\begin{array}{c}\text { Infiltrating } \\
\text { ductal carcinoma }\end{array}$ & $\begin{array}{l}\text { Infiltrating } \\
\text { lobular carcinoma }\end{array}$ & \\
\hline Positive & 1 & 1 & 3 & 4 & 1 & 1 & 7 & 5 & 23 \\
\hline Negative & 6 & 7 & 2 & 2 & 1 & 0 & 3 & 5 & 26 \\
\hline Total & 7 & 8 & 5 & 6 & 2 & 1 & 10 & 10 & 49 \\
\hline $\begin{array}{l}\text { Positive } \\
\text { rate }(\%)\end{array}$ & 14.29 & 12.50 & 60.0 & 66.7 & 50.0 & 100 & 70 & 50 & - \\
\hline
\end{tabular}

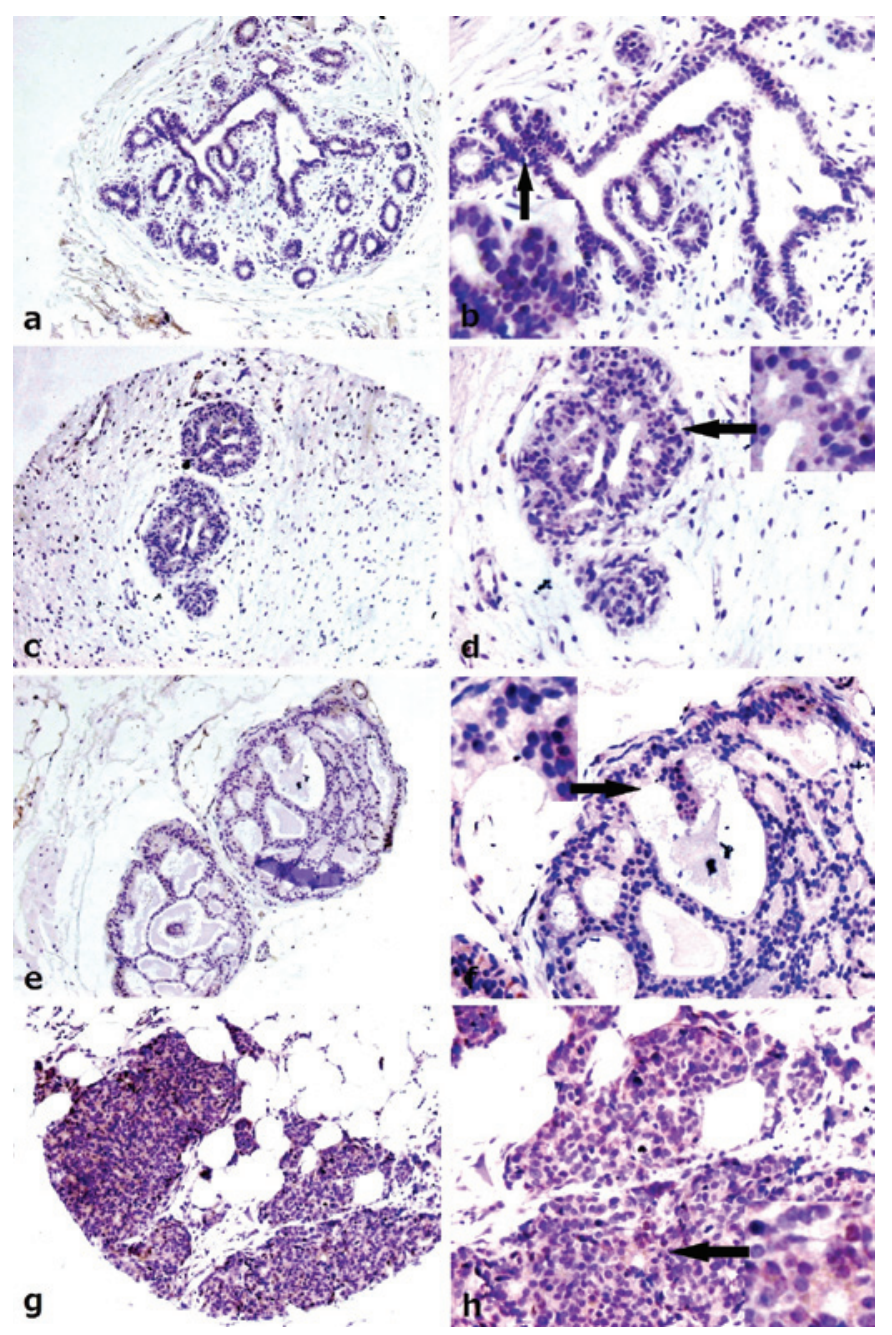

Figure 1. Expression of p-CREB-2 (ser 245) in tumor and non-tumor breast tissues using IHC and tissue microarray. Weak nuclear and cytoplasmic expression of p-CREB-2 (ser 245) in normal breast epithelia (a, x200; $\mathrm{b}, \mathrm{x} 400)$. Weak nuclear and cytoplasmic expression of p-CREB-2 (ser 245) in hyperplasia of breast epithelia (c, x200; d, x400). Scattered strong immunostaining of p-CREB-2 (ser 245) was observed in breast epithelia with dysplasia (e, x200; f, x400). Diffuse strong nuclear and cytoplasmic expression of p-CREB-2 (ser 245) in infiltrating ductal carcinoma of the breast (g, x200; h, x400). IHC, immunohistochemistry.

difference between each other $(\mathrm{p}>0.05)$. The positive rates in the three types of dysplasia (mild type: $60 \%, 3 / 5$; moderate type: $66.67 \%, 4 / 6$; severe type: $50 \%, 1 / 2$ ) displayed significant increases compared with the normal tissue and hyperplasia of breast epithelia $(\mathrm{p}<0.05)$, although no significant difference was achieved between the three types of dysplasia of breast epithelia $(\mathrm{p}>0.05)$. The positive rate of $\mathrm{p}-\mathrm{CREB}-2$ (ser 245) expression in carcinoma in situ was $100 \%$ yet the case number was too small to achieve a significant difference when compared with the other histological types. Immunostaining of p-CREB-2 (ser 245) was detected in 7 out of 10 cases of infiltrating ductal carcinoma and the positive rate $(70 \%)$ was significantly higher than that in normal epithelia, hyperplasia and dysplasia of the breast $(\mathrm{p}<0.05)$. The positive rate of p-CREB-2 (ser 245) expression in infiltrating lobular carcinoma was $50 \%$ (5/10), which was also higher than that in normal epithelia, hyperplasia and dysplasia of the breast $(\mathrm{p}<0.05)$. Immunofluorescent staining confirmed weak nuclear and cytoplasmic p-CREB-2 (ser 245) expression in normal breast epithelia MCF 10A cells and moderate to strong nuclear and cytoplasmic p-CREB-2 (ser 245) expression in breast cancer MCF 7 and MDA-MB-231 cells (Fig. 2). Western blotting revealed elevated expression of p-CREB-2 (ser 245) in breast carcinoma compared with normal breast tissues (Fig. 3) (Table II) (p<0.05).

Relationship between the expression of p-CREB-2 (ser 245) and clinicopathological factors in infiltrating breast carcinoma. The relationship between the expression of p-CREB-2 (ser 245) and various clinicopathological factors in infiltrating breast carcinoma are outlined in Table III. No significant correlation was found between the expression of p-CREB-2 (ser 245) and age, grade, T stage and TNM stage in the patients with infiltrating breast carcinoma ( $p>0.05)$. A significant association was noted between expression of p-CREB-2 (ser 245) and lymph node metastasis status. Higher expression of p-CREB-2 (ser 245) was noted in infiltrating breast carcinoma with lymph node metastasis $(\mathrm{p}<0.05)$.

\section{Discussion}

CREB2, also known as ATF4, belongs to a large family of basic region/leucine zipper (bZIP) transcription factors (1). These proteins bind to DNA via their basic region and dimerize via their leucine zipper domain to form a large variety of homodimers and/or heterodimers, which allow 


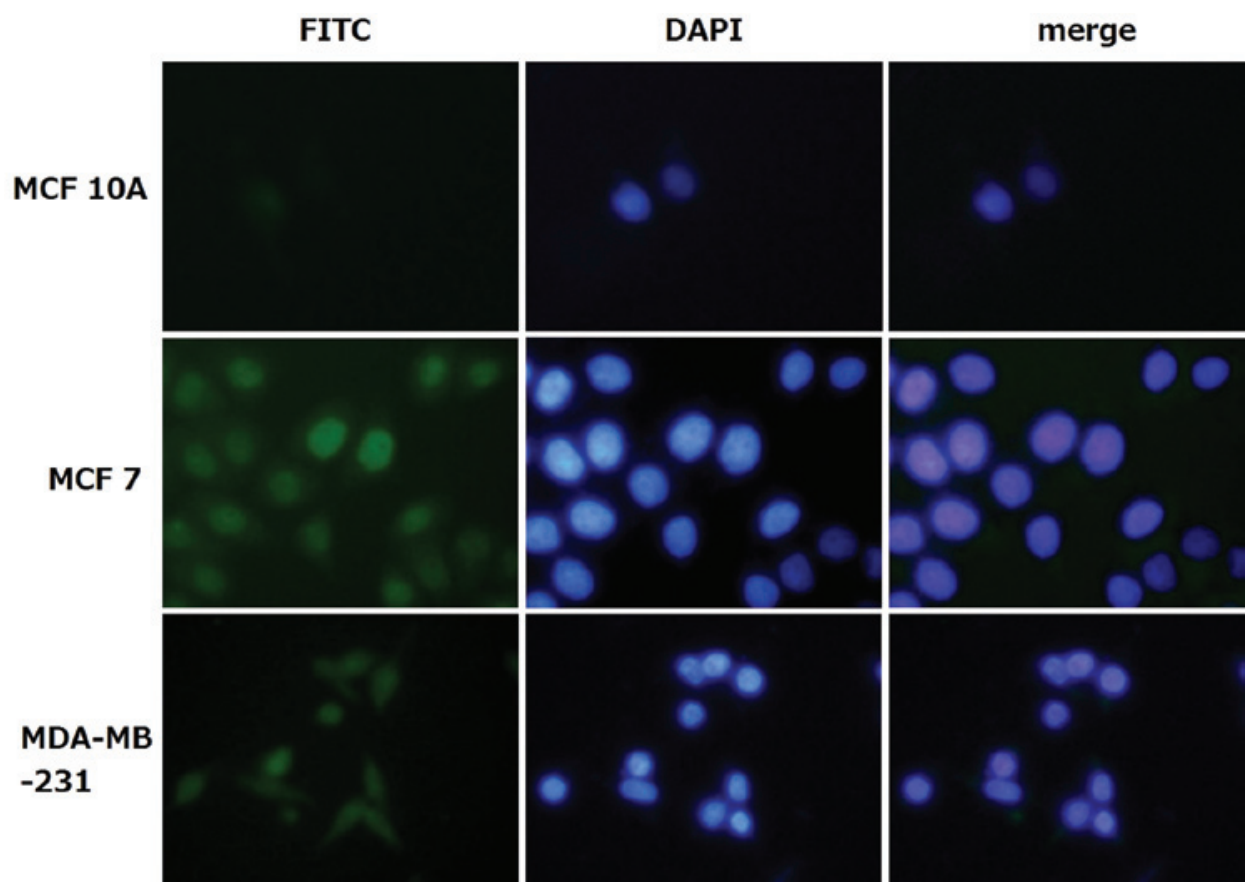

Figure 2. Immunofluorescent staining of p-CREB-2 (ser 245) in normal breast epithelial and cancer cell lines. Normal epithelial MCF 10A cells exhibited very weak nuclear staining of p-CREB-2 (ser 245). MCF 7 and MDA-MB-231 cancer cells exhibited strong nuclear and moderate cytoplasmic staining of p-CREB-2 (ser 245). DAPI, 4',6-diamidino-2-phenylindole; FITC, fluorescein isothiocyanate.

\section{N1 T1 N2 T2 N3 T3 N4 T4}

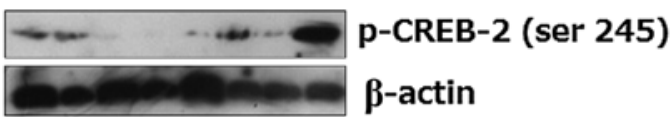

Figure 3. The Western blot analysis shows that compared to normal breast tissues $(\mathrm{N})$ there was elevated expression of p-CREB-2 (ser 245) (38 kDa) in breast carcinoma tissues $(\mathrm{T}) . \beta$-actin $(43 \mathrm{kDa})$ was used as an internal control.

the cell to coordinate signals from various pathways $(1,10)$. CREB-2 is widely expressed in a variety of tissues and tumor cell lines at the mRNA level but in the majority of cells the ATF4 protein is almost undetectable due to a very short halflife and low translation efficiency (11). Using human normal cell lines and Western blotting, Yang et al found that ATF4 is detectable specifically in osteoblast cell lines (12) while in the present study, we found that there was generally very weak expression of p-CREB-2 (ser 245) in normal breast tissues. For some cases, the protein bands of Western blotting were quite marked. The difference between our findings and those of Yang et al may be due to the samples used, as tissue samples are much more of a mixture compared to cell line samples. There are increasing data to indicate that ATF4 may be a stress-response protein and, consequently, may function as a regulator of the adaptive response to such stress $(2,3,11,13)$. In the present study, we found that, compared to normal breast tissues, the expression level of p-CREB-2 (ser 245) in breast cancer tissues was significantly elevated. Due to inefficient vascularization, increased diffusion distances exist in developing solid tumors, which often result in an insufficient supply
Table II. p-CREB-2 (ser 245) expression as detected by Western blotting in 17 pairs of normal and carcinoma breast tissues (t-test analysis).

\begin{tabular}{lccc}
\hline & $\begin{array}{c}\text { p-CREB-2 }(\text { ser } 245) \\
\text { expression }^{\mathrm{a}}\end{array}$ & $\mathrm{t}$ & $\mathrm{p}$-value \\
\hline Normal breast & $0.132 \pm 0.115$ & & \\
Cancer & $0.630 \pm 0.337$ & 6.060 & 0.000 \\
\hline
\end{tabular}

${ }^{\text {a } V a l u e s ~ w e r e ~ e x p r e s s e d ~ a s ~ t h e ~ m e a n ~} \pm \mathrm{SD}$.

of $\mathrm{O}_{2}$ and nutrients to rapidly expanding tissues. Increased levels of CREB-2 protein in breast cancer tissues may be due to the common hypoxia status in this solid tumor, although the mechanism involved in the upregulation of CREB-2 expression remains to be further studied.

In certain studies ATF4 was found to be essential for cell proliferation, particularly in human cancer cells $(13,14)$. CREB-2 was previously found to be upregulated in certain malignant tumors including breast carcinoma compared to their non-tumor counterparts (2); however, functions of this protein relative to tumor biology are poorly understood. In the present study, we focused on the expression of the phosphorylated form of CREB-2 at the 245 serine site in a group of breast tissues and cell lines. Elefteriou et al found that ATF4 phosphorylation on serine 254 increased PKA activity and eventually promoted osteoclast differentiation and bone resorption in mice (15). A study by Hogan et al indicated that phosphorylation of CREB-2 on serine 254 led to the signifi- 
Table III. Correlation between expression of p-CREB-2 (ser 245) and clinicopathological factors of the infiltrating breast carcinoma samples.

\begin{tabular}{|c|c|c|c|c|}
\hline \multirow[b]{2}{*}{ Variables } & \multirow[b]{2}{*}{ Total patients } & \multicolumn{2}{|c|}{ p-CREB-2 $(\operatorname{ser} 245)^{\mathrm{a}}$} & \multirow[b]{2}{*}{$\mathrm{p}$-value ${ }^{\mathrm{a}}$} \\
\hline & & Negative & Positive & \\
\hline Total & 20 & 8 & 12 & \\
\hline \multicolumn{5}{|l|}{ Age (years) } \\
\hline$<50$ & 11 & 4 & 7 & 0.714 \\
\hline$\geq 50$ & 9 & 4 & 5 & \\
\hline \multicolumn{5}{|l|}{ Grade } \\
\hline I & 8 & 3 & 5 & 0.413 \\
\hline II & 9 & 5 & 5 & \\
\hline III & 3 & 0 & 2 & \\
\hline \multicolumn{5}{|l|}{ TNM stage } \\
\hline I and II & 14 & 5 & 8 & 0.829 \\
\hline III and IV & 6 & 2 & 4 & \\
\hline \multicolumn{5}{|l|}{ T stage ${ }^{b}$} \\
\hline $\mathrm{T} 2$ & 12 & 5 & 7 & 0.692 \\
\hline $\mathrm{T} 3$ & 1 & 0 & 1 & \\
\hline $\mathrm{T} 4$ & 6 & 2 & 4 & \\
\hline \multicolumn{5}{|l|}{$\begin{array}{l}\text { Lymph node } \\
\text { metastasis }\end{array}$} \\
\hline Yes & 8 & 1 & 7 & 0.040 \\
\hline No & 12 & 7 & 5 & \\
\hline
\end{tabular}

${ }^{\mathrm{a}} \mathrm{p}$-values were obtained with the $\chi^{2}$ test. ${ }^{\mathrm{b}}$ Data for one sample is not included. There were no cases with T1

cant increase of CRE-reporter activation by the ATF4-ZF complex (7). In the present study, we attempted to discover whether p-CREB-2 (ser 245) has an impact on carcinogenesis and tumor biology of breast cancer. In this study, we found that there was very low expression of p-CREB-2 (ser 245) in normal breast epithelia, while increased expression was found in precancerous and cancer tissues, with the highest level detected in ductal breast carcinoma. The tendency of increasing expression of this phosphorylated form of the protein was consistent with that of total expression of this protein in breast cancer in a study by Ameri et al (2). From these results we can confer that at least part of the increasing protein in breast cancer is a result of this phosphorylated form and that this may also contribute to the carcinogenesis of breast carcinoma. Phosphorylation of ATF4 was found to occur at various sites by different kinases and to have varying functions. Yang et al showed that ATF4 phosphorylation on serine 251 in $\mathrm{Nf}^{-/-}$osteoblasts increased collagen synthesis and osteocalcin expression (6). Lassot et al found that serine 219 of ATF4 is required for interaction with bTrCP and subsequent proteasome-mediated protein degradation (16). However, to date, functions of the phosphorylated forms of the protein in normal and cancer cells are not yet clear. In the present study, we found that expression of p-CREB-2 (ser 245) increases from precancerous lesions to breast carcinoma, which indicates its potential roles in breast carcinogenesis, although the relative mechanism remains unclear. Studies by Roybal et al (13) and Zhang et al (14) revealed that CREB-2 is essential for cell proliferation, particularly in human cancer cells. In our study, the highest level of p-CREB-2 (ser 245) was detected in breast carcinoma compared with normal epithelia and precancerous lesions. Although the mechanism of this upregulated expression remains unknown, it is reasonable to assume that the phosphorylated form of this protein may play significant roles in the maintanance of the malignant form.

Recently, increasing data prove that CREB-2 may contribute to the hypoxic response $(2,3,11,13)$. It is well known that hypoxia perturbs and reduces protein folding capacity, resulting in the accumulation and aggregation of misfolded proteins in the lumen of the endoplasmic reticulum (ER). ATF4 has been found to be one of the main transcriptional regulators of the ISR and activates various UPR target genes that promote restoration of normal ER function. Previous studies have found that the PERK-ATF4 pathway acts predominantly as a pro-survival, rather than pro-death, pathway $(17,18)$. Recently, CREB-2 was also found to contribute to the chemotherapy resistance of cancer cells. Fung et al found that CREB-2 was capable of inducing DNA repair and anti-mutation responses to DNA damage induced by arsenite, which was used in therapy for acute promyelocytic leukemia (19). Tanabe et al found that cisplatin induced expression levels of CREB-2 mRNA and that ATF4-deficient cells were more sensitive to cisplatin-induced cell death (20). When we investigated the association of the expression level of p-CREB-2 (ser 245) to tumor biology, in particular the TNM stage of the tumor, we discovered that increased expression of p-CREB-2 was associated with lymph node metastasis. Although we are unaware as to whether this phosphorylated form contributes to the possible role of this gene in the hypoxic response and resistance to therapy, from the data here, we can confer that this phosphorylated protein may play significant roles in tumor development. To the best of our knowledge, no research exists that proves that CREB-2 is linked to tumor invasion. However, this finding is consistent with that of Afonyushkin et al, that CREB-2 may play critical roles in stress-induced angiogenesis through VEGF signaling, as angiogenesis is believed to favor tumor cell metastasis (21). Although there was no connection between p-CREB-2 (ser 245) expression and the $T$ stage of tumors in this study, which may be due to the relatively small cohort studied as CREB-2 has been found to be involved in the hypoxic response in solid tumors, it is possible that CREB-2 may be upregulated during tumor growth, which is often accompanied by escalated hypoxia. Thus, another possible explanation for our results may be that the tumor growth itself, which could be accompanied by elevated CREB-2 expression, may favor tumor metastasis, including lymph node invasion.

Based on the present findings, the phosphorylated form of CREB-2 at the ser 245 site was upregulated in breast carcinoma compared to normal epithelia and precancerous lesions of the breast, and may play crucial roles in carcinogenesis and tumor development of breast cancer. These findings provide further insight into the understanding of the functions of this 
protein relative to tumor biology and justify consideration of this gene for use in therapeutic interventions.

\section{References}

1. Karpinski BA, Morle GD, Jodi H, et al: Molecular cloning of human CREB-2: an ATF/CREB transcription factor that can negatively regulate transcription from the cAMP response element. Proc Natl Acad Sci USA 89: 4820-4824, 1992.

2. Ameri K, Lewis CE, Raida M, et al: Anoxic induction of ATF-4 through HIF-1-independent pathways of protein stabilization in human cancer cells. Blood 103: 1876-1882, 2004.

3. Oskolkova OV, Afonyushkin T, Leitner A, et al: ATF4-dependent transcription is a key mechanism in VEGF up-regulation by oxidized phospholipids: critical role of oxidized sn-2 residues in activation of unfolded protein response. Blood 112: 330-339, 2008.

4. Gjymishka A, Palii SS, Shan J, et al: Despite increased ATF4 binding at the C/EBP-ATF composite site following activation of the unfolded protein response, system A transporter 2 (SNAT2) transcription activity is repressed in HepG2 cells. J Biol Chem 283: 27736-27747, 2008.

5. Xuan B, Qian Z, Torigoi E, et al: Human cytomegalovirus protein pUL38 induces ATF4 expression, inhibits persistent JNK phosphorylation, and suppresses endoplasmic reticulum stress-induced cell death. J Virol 83: 3463-3474, 2009.

6. Yang X, Matsuda K, Bialek P, et al: ATF4 is a substrate of RSK2 and an essential regulator of osteoblast biology; implication for Coffin-Lowry syndrome. Cell 117: 387-398, 2004.

7. Hogan MR, Cockram GP and Lu R: Cooperative interaction of Zhangfei and ATF4 in transactivation of the cyclic AMP response element. FEBS Lett 58: 58-62, 2006.

8. Fattaneh AT and Peter D: World Health Organization Classification of Tumors: Pathology and Genetics: Tumors of the breast and Female genital organs. Hamilton SR and Aaltonen LA (eds). IARC Press, Lyon, 2004.

9. Carlson RW, Allred C, Anderson BO, et al: NCCN guidelines for patients: breast cancer, 2010.

10. Hewes RS, Schaefer AM and Taghert PH: The cryptocephal gene (ATF4) encodes multiple basic-leucine zipper proteins controlling molting and metamorphosis in Drosophila. Genetics 155: 1711-1723, 2000
11. Rzymski T, Milani M, Singleton DC, et al: Role of ATF4 in regulation of autophagy and resistance to drugs and hypoxia. Cell Cycle 8: 3838-3847, 2009.

12. Yang X and Karsenty G: ATF4, the osteoblast accumulation of which is determined post-translationally, can induce osteoblast-specific gene expression in non-osteoblastic cells. J Biol Chem 279: 47109-47114, 2004.

13. Roybal CN, Hunsaker LA, Barbash O, et al: The oxidative stressor arsenite activates vascular endothelial growth factor mRNA transcription by an ATF4-dependent mechanism. J Biol Chem 280: 20331-20339, 2005.

14. Zhang X, Yu S, Galson DL, et al: Activating transcription factor 4 is critical for proliferation and survival in primary bone marrow stromal cells and calvarial osteoblasts. J Cell Biochem 105: 885-895, 2008.

15. Elefteriou F, Benson MD, Sowa H, et al: ATF4 mediation of NF1 functions in osteoblast reveals a nutritional basis for congenital skeletal dysplasiae. Cell Metab 4: 441-451, 2006.

16. Lassot I, Emmanuel S, Clarisse BT, et al: ATF4 degradation relies on a phosphorylation-dependent interaction with the SCFbTrCP ubiquitin ligase. Mol Cell Biol 21: 2192-2202, 2001.

17. Koumenis C, Naczki C, Koritzinsky M, et al: Regulation of protein synthesis by hypoxia via activation of the endoplasmic reticulum kinase PERK and phosphorylation of the translation initiation factor eIF2alpha. Mol Cell Biol 22: 7405-7416, 2002.

18. Harding HP, Zhang Y, Bertolotti A, et al: Perk is essential for translational regulation and cell survival during the unfolded protein response. Mol Cell 5: 897-904, 2000.

19. Fung H, Liu $P$ and Demple B: ATF4-dependent oxidative induction of the DNA repair enzyme Apel counteracts arsenite cytotoxicity and suppresses arsenite mediated mutagenesis. Mol Cell Biol 27: 8834-8847, 2007.

20. Tanabe M, Izumi $\mathrm{H}$, Ise $\mathrm{T}$, et al: Activating transcription factor 4 increases the cisplatin resistance of human cancer cell lines. Cancer Res 63: 8592-8595, 2003.

21. Afonyushkin T, Oskolkova OV, Philippova M, et al: Oxidized phospholipids regulate expression of ATF4 and VEGF in endothelial cells via NRF2-dependent mechanism: novel point of convergence between electrophilic and unfolded protein stress pathways. Arterioscler Thromb Vasc Biol 30: 1007-1013, 2010. 